\title{
Genetic and Biochemical Effects of Natural Extracts on in Vitro Maturation of Egyptian Buffalo Oocytes
}

\author{
Ibrahim Abdalla Hassan Barakat ${ }^{1}$, Aziza Mohamed Hassan ${ }^{2 *}$, \\ Sally Said Alam² and Wagdy Khalil Bassaly Khalil ${ }^{2}$ \\ ${ }^{1}$ Zoology Department, College of Science, King Saud University, Riyadh, Saudi Arabia \\ ${ }^{2}$ Cell Biology Department, National Research Center, Dokki, Giza, Egypt
}

Received February 17, 2010; accepted July 3, 2010

\begin{abstract}
Summary Preparation of oocytes is one of the critical factors that determine the developmental competence of embryos produced by in vitro fertilization (IVF). The present study was conducted to evaluate the effect of different maturation media on the nuclear and cytoplasmic maturation of buffalo oocytes. Also, to investigate if the addition of ginseng and fenugreek to the maturation media during in vitro maturation (IVM) would influence on IVM, and developmental competence of buffalo oocytes as well as intracellular GSH level. Three experiments were conducted to achieve the goals of present study. In the first experiment, the buffalo oocytes were cultured in different media without any supplementations to detect the most suitable effective medium for IVM. The second experiment compared between different culture media for IVM of oocytes through measurement of intracellular glutathione level. The third experiment examined the effect of ginseng and fenugreek additives during the IVM of Egyptian buffalo oocytes. The results of this study proved that the TCM-199 medium significantly increased the percentage of cumulus expansion, nuclear maturation and intracellular GSH concentration after IVM of buffalo oocytes, while CR1aa was the least effective medium. Treatment with ginseng and fenugreek extracts during IVM culture efficiently improves the development competence of buffalo oocytes, and this improvement is correlated with increase of intracellular GSH concentration. In conclusion, TCM-199 was the most effective medium and is desirable for in vitro maturation of Egyptian buffalo oocytes. Ginseng and fenugreek additives enhanced the quality of less effective medium (CR1aa), as well as optimized the effect of TCM-199 medium.
\end{abstract}

Key words Buffalo, IVM, Cytogenetic, Glutathione, Fenugreek, Ginseng.

Water buffalo (Bubalus Bubalis) is a distinct species within the family Bovidae and it has a distinguished position in the Egyptian agriculture system. Decreases in the buffalo population, as well as increases in the demand for buffalo meat, have led to urgent requirements for the improvement of breeding strategies and the genetics of this domestic livestock. Modern reproductive biotechnologies, such as embryo transfer and in vitro embryo production (IVEP), have been used as adjuncts to conventional breeding programs, with limited success (Palta and Chauhan 1998). Despite this intrinsic limitation, improvements are possible through the optimization of the different steps of the procedures that can be achieved by taking into account species-specific differences, as indicated by the higher blastocyst yields reported over recent years (Gasparrini et al. 2006). Major factors that might affect the IVEP efficiency are inadequate oocyte maturation, an inappropriate timing of insemination and a non-optimal time of gamete co-incubation.

Oocyte developmental competence, an important factor that affected the IVEP, which involves the ability of a germ cell to produce a normal and viable embryo after fertilization, is a condition that results from both nuclear and cytoplasmic maturation (Niemann and Rath 2001). Many factors influence the karyoplasmic and cytoplasmic maturation of oocytes in vitro, including co-culturing

\footnotetext{
*Corresponding author, e-mail: Dr_azizahassan@yahoo.com, azizayahassan@yahoo.com
} 
with follicular cells such as cumulus cells or granulose cells, duration of maturation, and type of media (de Matos and Furnus 2007, Dilip et al. 2006). The process of oocyte cytoplasmic maturation involves numerous molecular events, including synthesis of biochemical compounds, protein phosphorylation and activation of particular metabolically pathways (Eppig 1996, Krisher and Bavister 1999). The synthesis of intracellular glutathione (GSH) is a critical part of oocyte cytoplasmic maturation (Eppig 1996). In bovine oocytes, synthesis of GSH during IVM has been reported (Miyamura et al. 1995, de Matos et al. 2002), and GSH participates in the male pronucleus formation after fertilization (Miyamura et al. 1995, de Matos et al. 1996). Therefore, GSH is a potentially highly relevant biochemical marker of the viability and developmental capacity of mammalian oocytes (Zuelke et al. 2003). However, the developmental ability of oocytes matured in defined media still tends to be lower than that of oocytes matured in media supplemented with chemicals such as amino acids or antioxidants (Herrick et al. 2004, Hong et al. 2004).

The maturation medium, selection of protein supplements, and hormones for IVM play an important role in the subsequent fertilization and development of mammalian oocytes during in vitro culture (Bavister and Rose-Hellekant 1992). However, the addition of steroids (estrogen and progesterone) improves the completion of maturation (Moor et al. 1980) and plays an important role not only for nuclear maturation but also for cytoplasmic maturation of sheep (Guler et al. 2000), cattle (Ali and Sirard 2002) and human oocytes (Tesarik and Mendoza 1997).

Phytoestrogens are natural plant substances similar to endogenous estrogens by their chemical structure. Due to this similarity they can bind to estrogen receptors (Cassidy 2003). Fenugreek (Trigonella foenum graecum) is one such plant whose termed phytoestrogens, its seeds and leaves are used not only as food but also as an ingredient in traditional medicine. Its seeds contain diosgenin which is used in synthetic estrogen (Billaud 2001). Diosgenin, a steroid sapogenin, is the starting compound for over $60 \%$ of the total steroid production by the pharmaceutical industry (Jayaweera 1981, Petit et al. 1995). Administration with fenugreek extract significantly increased the total number of the oocytes as well as improved the quality of oocytes in mice (Hassan et al. 2006). A steroid sapogenin constituent of fenugreek seeds is a precursor of steroid hormones, such as progesterone and anti-inflammatory steroids, such as cortisone (Norton 1998).

Ginseng is a popular herb with a long history of medicinal use; ginseng roots contain various pharmaceutical components, ginsenosides are the most pharmaceutically active (Kim et al. 2005). Traditionally, ginseng is considered a safe herb with few adverse effects. Estrogen-like effects after the use of ginseng products have been reported (Palop et al. 1999). Patients treated with ginseng extract had increased the total and free plasma follicle-stimulating hormone (FSH), and luteinizing hormone (LH) level (Tsai et al. 2003) but decreased prolactin levels (Salvati et al. 1996). Similarity of the ginseng molecule to the structure of some steroid hormones may contribute to the plant's pharmacological actions (Gray et al. 2004). According to the available literature, there are no reports where the fenugreek or ginseng has been used as phyto-estrogenic agents on in vitro maturation of buffalo oocytes. The aims of the present study were to evaluate the effect of different maturation media on the nuclear and cytoplasmic maturation of buffalo oocytes. Also, to investigate the effect of ginseng and fenugreek supplementation to the maturation media during IVM on the in vitro maturation, developmental competence of buffalo oocytes as well as intracellular GSH level. To our knowledge, the present study is the first one to show the stimulatory effect of ginseng and fenugreek addition to maturation medium on the improvement of buffalo oocytes developmental competence. 
Materials and methods

\section{Reagents and media}

All the chemicals were purchased from Sigma Chemical Co. (St. Louis, MO, USA) unless indicated otherwise. Folligon was purchased from Intervet International BV. (Boxmee-Holland). Fetal calf serum (FCS) (GIBCO, Grand Island, NY). The IVM media were; 1) TCM199 buffered with $25 \mathrm{mM}$ sodium bicarbonate; 2) Ham's F10 and 3) CR1aa (prepared as described by Rosenkrans and First 1994). All maturation media were supplemented with $40 \mathrm{IU} / \mathrm{ml}$ Folligon, $1 \mu \mathrm{g} / \mathrm{ml}$ estradiol- $17 \beta, 50 \mu \mathrm{g} / \mathrm{ml}$ gentamicin sulfates, and $10 \%(\mathrm{v} / \mathrm{v})$ fetal calf serum. Ginseng extract used in this study was standardized Panax ginseng extract EFLA400 (Phoenix ginseng) (Batch No. 303298) supplied from Lotte Group R \& D Centre (Seoul, Korea). Water extracts of fenugreek were presented as a yellow freeze dried crystalline form.

\section{Collection of oocytes and in vitro maturation}

Immediately after the slaughter of adult female buffalo, ovaries were collected and placed into Dullecco's phosphate buffered saline (DPBS) at $37^{\circ} \mathrm{C}$ and transported to the laboratory, within 2-3 h from slaughter. Cumulus-oocyte complexes (COCs) were recovered by aspiration of 2-8 mm follicles using a 20-gauge. The COCs were evaluated on the basis of their morphology. Only oocytes with compact layers of cumulus cells and intact cytoplasm were used for the experiments. The selected COCs were washed thoroughly in phosphate buffer saline (PBS) supplemented with $0.3 \%$ bovine serum albumin and once in the final maturation medium and cultured in the same medium. IVM was carried out at $38.5^{\circ} \mathrm{C}$ for $24 \mathrm{~h}$ in a controlled gas atmosphere of $5 \% \mathrm{CO}_{2}$ in humidified air. After maturation period, half of matured oocytes were frozen at $-80^{\circ} \mathrm{C}$ in microfuge tubes after removal of cumulus cells until the day of GSH assay. Each tube containing 20-30 oocytes in $5 \mu \mathrm{l}$ of PBS. The other half of matured oocytes was subjected to nuclear staining to evaluate the nuclear maturation rate.

\section{Cytogenetic studies}

At the end of the in vitro culture period, oocytes were firstly evaluated depending on expansion of cumulus cells, then transferred into $400 \mu \mathrm{l}$ of PBS solution and stripped from their cumulus cells by mechanical displacement by gentle mouth pipetting using a small-bore glass pipette. Matured oocytes were fixed with acetic alcohol (methanol and acetic acid, $3: 1, \mathrm{v} / \mathrm{v}$ ) for $24-48 \mathrm{~h}$ and stained with $1 \%$ aceto-orcein in $45 \%(\mathrm{v} / \mathrm{v})$ acetic acid. The stages of oocytes were classified according to Santos et al. (2006) as follows, intact germinal vesicle (GV; unidentifiable nucleolus and very fine filaments of chromatin); germinal vesicle breakdown (GVBD; presence of different patterns of chromatin condensation, chromosomes coiled up and no visible individual chromosomes); metaphase I (MI), anaphase I (AI), metaphase II (MII), once the formation of bivalents was completed and appeared on 1 or 2 sets of chromosomes.

\section{Glutathione assay}

Intracellular content of GSH was measured as described by Funahashi et al. (1994). The frozen samples were thawed at room temperature; this procedure was repeated three 3 . Five microliters of $0.2 \mathrm{M}$ sodium phosphate containing $10 \mathrm{mM}$ EDTA (Sigma, assay buffer) and $5 \mu \mathrm{l}$ of $1.25 \mathrm{M}$ phosphoric acid (Fluka, Buchs, Switzerland) were added to a $1.5 \mathrm{ml}$ microfuge tube containing 30 oocytes. Samples were stored at $-80^{\circ} \mathrm{C}$ until use. Concentrations of GSH in the oocytes were determined by the dithionitrobenzonic acid-glutathione disulphide (DTNB-GSSG) reductase-recycling assay (Anderson 1985). Briefly, $700 \mu \mathrm{l}$ of $0.33 \mathrm{mg} \mathrm{NADPH} / \mathrm{ml}$ in stock buffer, $100 \mu \mathrm{l}$ of $6 \mathrm{mM}$ DTNB in the stock buffer and $190 \mu \mathrm{l}$ water were added with mixing into the microfuge tube. To initiate the reaction, $10 \mu \mathrm{l}$ glutathione reductase was added with mixing. The 
formation of 5-thio-2-nitrobenzoic acid was followed continuously and the absorbance was monitored at $412 \mathrm{~nm}$ with a spectrophotometer (Ultraspec-2000, Pharmacia Biotech Ltd., Cambridge, UK) and recorded at $30 \mathrm{~s}$ and $5 \mathrm{~min}$. Both the reagent blank and GSH standards $(0.1-1.0 \mathrm{nmol})$ were assayed under the same conditions. The amount of GSH in each sample was determined by comparing it with a standard curve prepared at the same time.

\section{Experimental design}

Egyptian buffalo oocytes were in vitro matured, as described before, for $22-24 \mathrm{~h}$. After this period, samples of oocytes were examined for nuclear maturation stage and the rest of oocytes were processed for GSH assay. Replicates of experiments were performed on different days with different batches of oocytes.

Experiment 1 The first experiment was carried out to determine the effects of maturation media (TCM-199, Ham's F10 and CR1aa) on maturation rate depending on nuclear progression.

Experiment 2 The second experiment was carried out to measure the concentration of intracellular glutathione of buffalo oocytes matured in different culture media (TCM-199, Ham's F10 and CR1aa).

Experiment 3 This experiment was conducted based on the results obtained from experiments 1 and 2. In this experiment we studied the effect of the addition of $0.1 \mu \mathrm{g} / \mathrm{ml}$ (fenugreek or ginseng extracts) and $0.6 \mathrm{mM}$ cysteine, as a positive control, on the in vitro maturation media (TCM-199 and CR1aa) on maturation rate of buffalo oocytes with respect to cumulus expansion and intracellular glutathione. The nuclear progression of oocytes matured in less effective medium supplemented with the extracts was evaluated to confirm the improving effect of extracts.

\section{Statistical analysis}

Statistical analyses for all data were carried out using analysis of variance (ANOVA) and Duncan's test using the SPSS program. Data in the text were expressed as mean \pm SE. Analysis was used to compare the rate of maturation and GSH concentration in matured oocytes. A probability of $p<0.05$ was considered statistically significant.

\section{Results}

\section{Experiment 1: Effect of different maturation media on in vitro maturation of buffalo oocytes}

To determine the effect of different maturation media on the developmental competence of buffalo oocytes, we compared the proportions of matured oocytes and the cumulus expansion percentage after $24 \mathrm{~h}$ of IVM. The data concerning the influence of different maturation media on the percentage of cumulus expansion is shown in Figs. 1 and 2. The percentage of oocytes with expanded cumulus was highly significant $(p<0.5)$ in TCM-199 $(90 \%)$ medium when compared with that in CR1aa (82.5\%).

Regarding to the nuclear maturation, the results obtained in Table 1 showed that there were no significant differences in oocytes stages; GV, GVBD, AI, MI and MII; in TCM-199 and Ham' F10 media (Fig. 3). CR1 aa medium showed significantly difference when compared with TCM-199 and Ham's F10 media in all oocytes stages, with the exception in GV stage which did not show any significant difference between all media. The number of oocytes developed to MI and MII in TCM199 medium (30.6 \pm 2.8 and $26.0 \pm 2.9$, respectively) was significantly higher than those in CR1aa medium (16.6 \pm 1.2 and $18.2 \pm 1.2$, respectively). The percentage of nuclear maturation in TCM-199 and Ham's media (59\% and 46\%, respectively) was significantly higher compared to CR1aa medium (37\%). 
Experiment 2: Effect of different maturation media on intracellular GSH concentration in buffalo oocytes during IVM

Results of GSH concentrations are shown in Fig. 4 and revealed a significant increase in GSH levels in oocytes matured in TCM-199 medium (6.2 \pm 0.4 pmol/oocytes) compared to the other media; Ham's F10 and CR1aa (5.3 \pm 0.4 and $3.9 \pm 0.3$ pmol/oocytes, respectively).

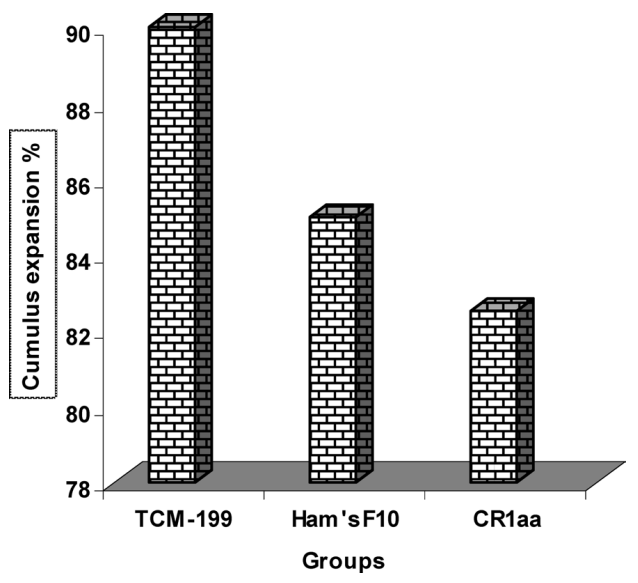

Fig. 1. Effect of different maturation media on the percentage of cumulus expansion of buffalo oocytes matured in vitro.

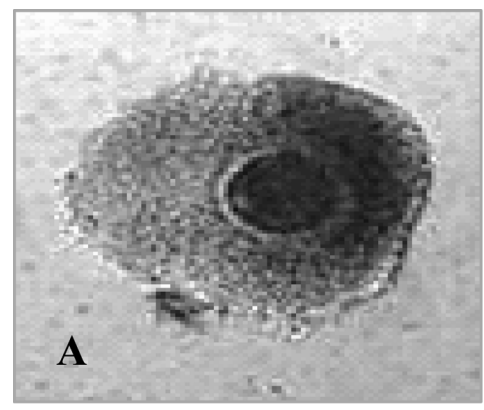

Experiment 3: Effect of fenugreek and ginseng extracts during IVM on oocytes maturation

The results obtained from the first and second experiments revealed that CR1aa medium was the least effective for maturation of buffalo oocytes while TCM-199 was most effective. So, the third experiment was conducted to determine the effects of treatment with fenugreek, ginseng extracts, and cysteine, as a positive control, in CR1aa and TCM-199 media on in vitro maturation of buffalo oocytes. As shown in Fig. 5 the concentration of intracellular GSH significantly increased in TCM-199 medium when supplemented with ginseng and fenugreek (8.8 \pm 0.3 and $7.8 \pm 0.3 \mathrm{pmol} /$ oocyte, respectively) compared

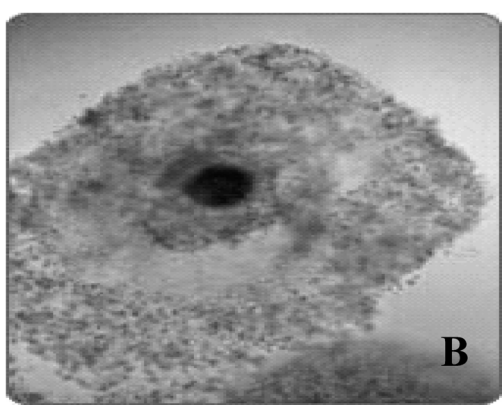

Fig. 2. Compact cumulus (A): tightly attached cells surrounding the oocyte with a smooth surface over the cumulus hillock. Expanded cumulus (B): cells detached from the oocyte with matrix visible between cumulus cells.

Table 1. Effects of different maturation media on nuclear maturation and meiotic progression of buffalo oocytes during IVM

\begin{tabular}{|c|c|c|c|c|c|c|c|}
\hline \multirow{2}{*}{ Groups } & \multirow{2}{*}{$\begin{array}{c}\text { No. of } \\
\text { oocytes }\end{array}$} & \multirow{2}{*}{$\begin{array}{c}\text { Nuclear } \\
\text { maturation } \\
\%\end{array}$} & \multicolumn{5}{|c|}{ Meiotic progression } \\
\hline & & & GV & GVBD & MI & AI & MII \\
\hline TCM-199 & 519 & $\begin{array}{c}305 / 519 \\
59 \%\end{array}$ & $6.2 \pm 1.7^{\mathrm{a}}$ & $19.6 \pm 1.1^{\mathrm{a}}$ & $30.6 \pm 2.8^{\mathrm{a}}$ & $6.6 \pm 0.5^{\mathrm{a}}$ & $26.0 \pm 2.9^{\mathrm{a}}$ \\
\hline Ham's F10 & 651 & $\begin{array}{c}302 / 651 \\
46 \%\end{array}$ & $7.2 \pm 0.5^{\mathrm{a}}$ & $18.2 \pm 1.1^{\mathrm{a}}$ & $28.0 \pm 3.0^{\mathrm{a}}$ & $5.4 \pm 0.5^{\mathrm{a}}$ & $25.4 \pm 1.2^{\mathrm{a}}$ \\
\hline CR1aa & 560 & $\begin{array}{c}200 / 560 \\
37 \%\end{array}$ & $6.2 \pm 0.5^{\mathrm{a}}$ & $13.2 \pm 0.6^{\mathrm{b}}$ & $16.6 \pm 1.2^{\mathrm{b}}$ & $2.2 \pm 0.7^{\mathrm{b}}$ & $18.2 \pm 1.2^{\mathrm{b}}$ \\
\hline
\end{tabular}

Data are presented as the mean \pm S.E.M. Values with different superscripts are significantly different $(p<0.05)$. 
Table 2. Effects of ginseng and fenugreek during IVM on nuclear maturation and meiotic progression in buffalo oocytes matured in CR1aa medium

\begin{tabular}{|c|c|c|c|c|c|c|c|}
\hline \multirow{2}{*}{ Groups } & \multirow{2}{*}{$\begin{array}{l}\text { No. of } \\
\text { oocytes }\end{array}$} & \multirow{2}{*}{$\begin{array}{l}\text { Nuclear } \\
\text { maturation } \\
\%\end{array}$} & \multicolumn{5}{|c|}{ Meiotic progression } \\
\hline & & & GV & GVBD & MI & AI & MII \\
\hline CR1aa & 560 & $\begin{array}{c}200 / 560 \\
37 \%\end{array}$ & $6.2 \pm 0.5^{\mathrm{ab}}$ & $13.2 \pm 0.58^{\mathrm{a}}$ & $16.6 \pm 1.2^{\mathrm{b}}$ & $2.2 \pm 0.7^{\mathrm{b}}$ & $18.2 \pm 1.2^{\mathrm{c}}$ \\
\hline CR1aa+Ginseng & 320 & $\begin{array}{c}167 / 320 \\
52.5 \%\end{array}$ & $5.6 \pm 0.75^{\mathrm{b}}$ & $8.6 \pm 1.5^{\mathrm{b}}$ & $20.0 \pm 3.5^{\mathrm{a}}$ & $3.9 \pm 0.51^{\mathrm{a}}$ & $28.6 \pm 0.68^{\mathrm{a}}$ \\
\hline CR1aa+Fenugreek & 370 & $\begin{array}{c}156 / 370 \\
41.8 \%\end{array}$ & $7.2 \pm 0.58^{\mathrm{a}}$ & $9.8 \pm 0.37^{b}$ & $19.0 \pm 1.2^{\mathrm{a}}$ & $3.0 \pm 0.89^{\mathrm{a}}$ & $26.2 \pm 0.58^{\mathrm{b}}$ \\
\hline CR1aa + Cysteine & 300 & $\begin{array}{c}129 / 300 \\
43 \%\end{array}$ & $6.6 \pm 0.81^{\mathrm{ab}}$ & $10.8 \pm 1.66^{\mathrm{ab}}$ & $15.6 \pm 1.47^{\mathrm{b}}$ & $1.4 \pm 0.51^{b}$ & $26.0 \pm 0.71^{\mathrm{b}}$ \\
\hline
\end{tabular}

Data are presented as the mean \pm S.E.M. Values with different superscripts are significantly different $(p<0.05)$.

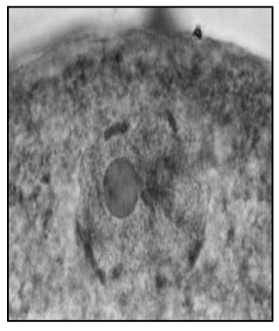

GV

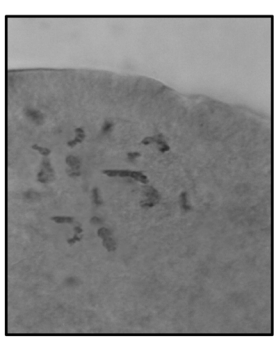

GVBD

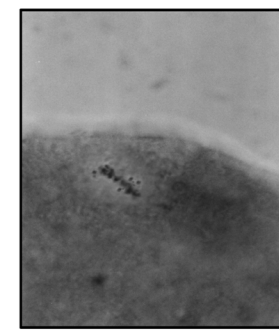

MI

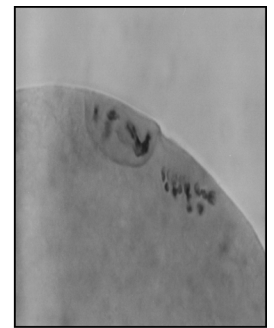

MII

Fig. 3. Photomicrographs of stained buffalo oocytes represent various nuclear stages during meiotic progression.

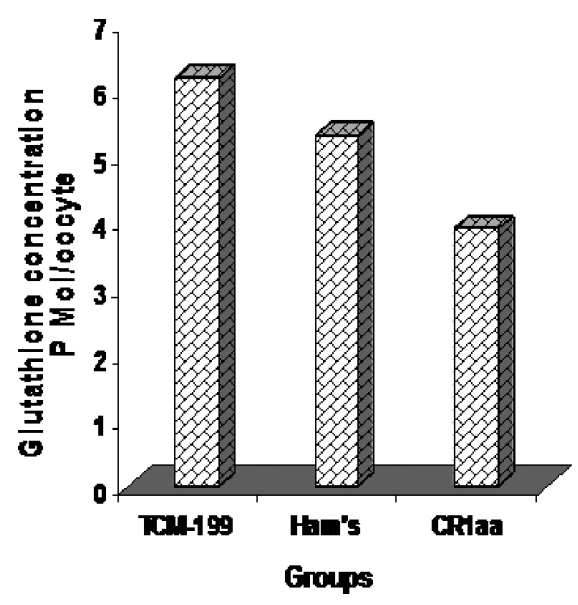

Fig. 4. Effect of different maturation media on the concentration of the intracellular GSH in buffalo oocytes during IVM. to those matured in TCM-199 medium without supplementation ( $6.0 \pm 0.4 \mathrm{pmol} /$ oocyte). Moreover, GSH concentrations were significantly increased in oocytes matured in CR1aa medium supplemented with ginseng and fenugreek $(6.0 \pm 0.28$ and $5.4 \pm 0.22 \mathrm{pmol} /$ oocyte, respectively) compared to those matured in CR1 aa medium alone $(3.9 \pm 0.31 \mathrm{pmol} /$ oocyte). Supplementation with ginseng and fenugreek during IVM significantly increased the concentration of GSH in oocytes matured in TCM-199 compared to CR1aa media.

The proportion of oocytes with expanded cumulus cells was significantly higher in all supplemented media compared to TCM-199 and CR1aa without supplementation $(p<0.5)$ as shown in Fig. 6. Ginseng and fenugreek supplementation to culture media during IVM increase the proportions of oocytes with expanded cumulus cells in TCM-199 than CR1aa medium. However, no significant difference was observed between TCM-199 and CR1 aa supplemented with cysteine.

The progression through the meiotic stages of buffalo oocytes matured in CR1aa medium 


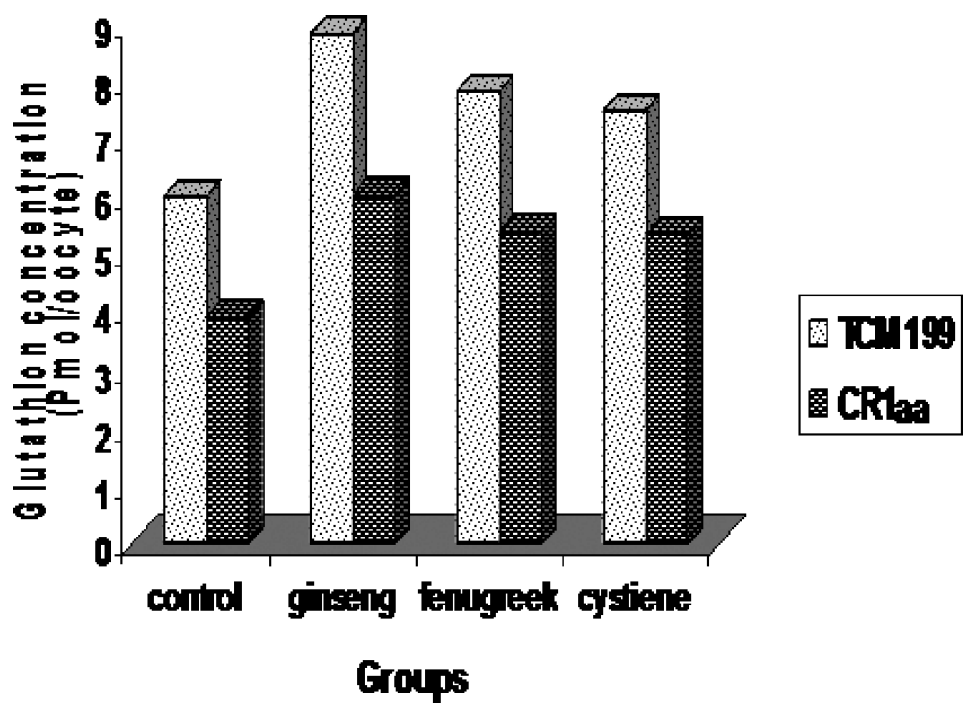

Fig. 5. Effect of ginseng and fenugreek addition to TCM-199 and CR1aa media on the concentration of the intracellular GSH in buffalo oocytes.

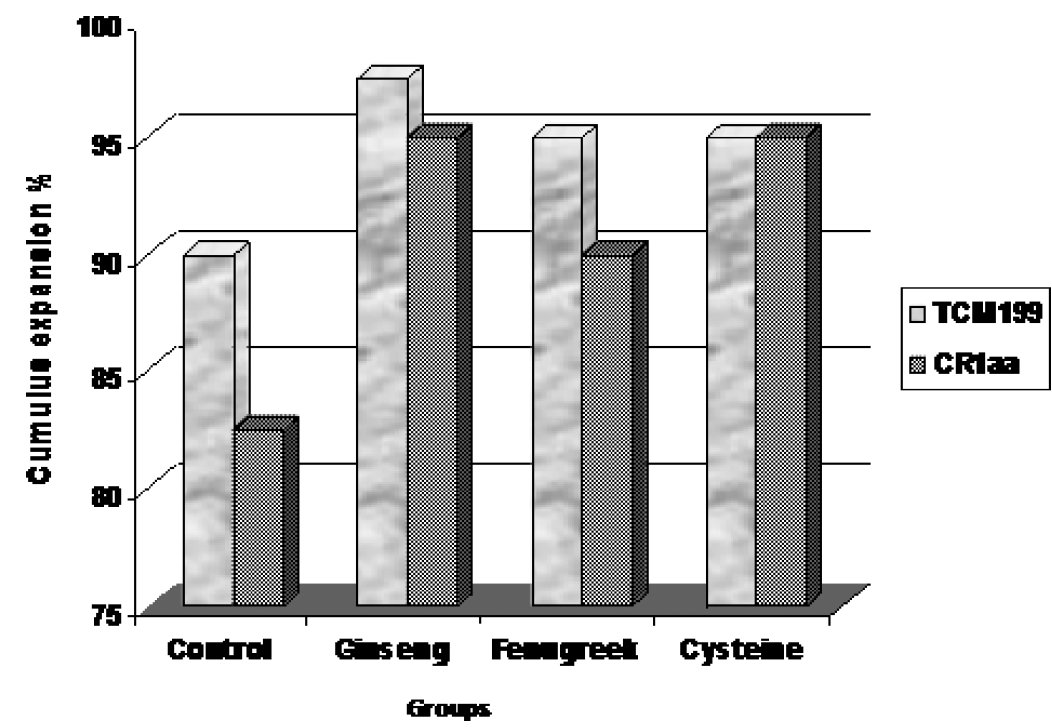

Fig. 6. Effect of ginseng and fenugreek addition to TCM-199 and CR1aa media on the percentage of cumulus expansion.

supplemented with ginseng, fenugreek and cysteine is presented in Table 2. The highest $(p<0.5)$ proportion of oocytes completed nuclear maturation (i.e. reached the MII stage) were observed in CR1aa plus ginseng compared to all other groups. Furthermore, all the supplemented media showed an increase in the rate of oocytes that reached the MII stage compared to the non-supplemented CR1aa medium.

\section{Discussion}

Development of a suitable culture system for IVM of oocytes is a major component of in vitro 
embryo production procedures, as it is not only affects the proportion of oocytes that reach the metaphase II, but also influences subsequent embryonic development (Rizos et al. 2002).

In this study, TCM-199, Ham's F-10 and CR1aa media were used for in vitro maturation of buffalo oocytes and were compared for their efficiency in enhancing both nuclear and cytoplasmic maturation. The results revealed that using the TCM-199 medium during IVM of buffalo oocytes significantly increased the nuclear maturation and induced greater cumulus expansion in comparison with Ham's F-10 and CR1aa media. Our results are in agreement with other studies, which also showed higher maturation rates in TCM-199 than in Ham's F-10 (Pawshe et al. 1996, Smetanina et al. 2000). Similar findings have been reported by Jamil et al. (2007), who found a significant difference for maturation rate of buffalo oocytes matured in both media. A variety of media have been used for in vitro maturation of follicular oocytes, however TCM-199 medium was the basic maturation medium in many recent studies (Li et al. 2004, Jamil et al. 2007, Amer et al. 2008). Roushandeh et al. (2007) found that TCM-199 medium improved GVBD and MII development better than MEME medium. TCM-199 has a lower glucose concentration, both essential and non-essential amino-acids, a large range of vitamins and several other components such as cholesterol and ribose. Amino acids are known to be beneficial for embryo development, and oocyte maturation (Ruth et al. 2002).

Glutathione plays many important biological roles and it has been suggested that GSH synthesis is critical for the acquisition of developmental competence of oocytes at the cytoplasmic level (Eppig 1996). This is reinforced by the observation that the intracellular GSH concentration of porcine oocytes following IVM reflects the degree of cytoplasmic maturation (Funahashi et al. 1994). Furthermore, de Matos and Furnus (2000) suggested that the measurement of GSH levels at the end of IVM may represent a reliable indicator of the cytoplasmic maturation and its concentration could be affected by maturation madia (Yoshida et al. 1993). In the present study, the highest intracytoplasmic GSH concentration was found in oocytes matured in TCM-199 medium followed by Ham's F-10 then CR1aa. These findings are consistent with the report of Yoshida et al. (1992) who reported that glutathione level in pig oocytes matured in TCM-199 was significantly higher than those matured in TLP-PVA and the finding of Hibasami et al. (2003) who concluded that TCM-199 is the basic maturation medium used in the in vitro oocytes maturation.

Among its other multiple roles, previously described, glutathione is also critically involved in the cellular defense against oxidative stress, which is one of the most important factors interfering with a proper cell function (Gasparrini et al. 2003). The addition of antioxidants to IVM media significantly increases the glutathione content and improved the developmental competence of oocytes (Wang et al. 2007). The present study demonstrated that the addition of ginseng and fenugreek to TCM-199 and CR1aa maturation media during IVM significantly increased the level of GSH compared with control media (non-supplemented groups) and cysetine supplemented groups, which indicated that the improvement of developmental competence of buffalo oocytes may be partly due to increase of intracellular GSH concentration after IVM of oocytes. Several authors have postulated that the measurements of GSH concentration in oocytes after IVM may be a valuable indicator of the oocyte cytoplasmic maturation in bovine (de Matos and Furnus 2000), ovine (de Matos et al. 2002) and caprine (Cognié et al. 2003). In addition, fenugreek leaves are a rich source of calcium, iron, B-carotene and other vitamins (Sharma et al. 1996) which have been found to have antioxidant and anticarcinogenic potency in different settings (Sur et al. 2001, Hibasami et al. 2003). Moreover, ginseng roots contain various pharmaceutical components like ginsenosides (saponins), polyacetylenes and polyphenolic compounds which have strong antioxidant activity (Higdon and Frei 2003, Kim et al. 2005). Hassan and Abdel-Wahhab (2006) reported that ginseng has an antioxidant effect and improved sperm quality in mice. Both fenugreek and ginseng have antioxidant activity, and it may be attributed to stimulate synthesis of glutathione in oocytes during IVM. 
In this study, the addition of ginseng or fenugreek extracts to CR1aa medium significantly enhanced the maturation rate. This finding suggested that the enhancement in the maturation rate may be attributed to the steroid compound in both extracts. In IVM, the addition of steroids improved the completion of maturation (Moor et al. 1980). However, in vivo the balance between the gonadotropins (FSH and LH) and steroids (estrogen and progesterone) might be the important criteria in the initiation of follicle growth and good-quality oocytes (Amer et al. 2008). Administration with fenugreek extract significantly increased the total number of the oocytes as well as improved the quality of oocytes in mice (Hassan et al. 2006). Fenugreek seeds contain diosgenin which is used to make synthetic estrogen (Billaud 2001). Diosgenin, a steroid sapogenin, is the initial compound for over $60 \%$ of the total steroid production by the pharmaceutical industry (Jayaweera 1981, Petit et al. 1995). While the similarity of the ginsenoside molecule, in ginseng, to the structure of some steroid hormones may contribute to the plant's pharmacological actions (Gray et al. 2004). In another study the interaction of this ginsenoside with the estrogen receptor was reported (Lee et al. 2003). Also estrogen-like effects after use of ginseng products have been reported by Palop et al. (1999). Our results demonstrated that the addition of ginseng extract during IVM had a significantly higher $(p<0.05)$ effect on maturation rate than fenugreek extract. Oocytes matured in vitro in the presence of gonadotrophins and steroid had higher maturation rates compared with maturation protocols, in which no hormones were added (Choi et al. 2001). Gonadotrophins and steroid cause synergistic enhancement of nuclear maturation in mammalian oocytes in vitro (Pawshe et al. 1996). Also, oocytes matured in vitro in the presence of gonadotrophins and steroid especially estradiol showed higher maturation and fertilization rates compared with those matured in the absence of hormones (Abdoon et al. 2001). Hence, supplementation of the media with ginseng extract had a biphasic favorable effect on maturation.

In conclusion, the results of the current study showed that TCM-199 medium was most effective during IVM of buffalo oocytes while CR1aa was less effective one. Addition of ginseng or fenugreek extracts during IVM efficiently improves the development competence of buffalo oocytes, and this improvement is correlated with increase of intracellular GSH concentration after IVM of oocytes. Ginseng and fenugreek enhanced the quality of CR1aa medium as well as optimized the effect of TCM-199 medium. Use of alternative concentrations and culture conditions to manipulate supplementation of culture media with natural extracts will be useful in further elucidating the mechanisms of these actions. This initial hypothesis needs further proof, but it may explain why different phyto-estrogens exert a more or less inhibitory influence on the maturation processes of oocytes.

\section{References}

Abdoon, A., Kandil, O., Otio, T. and Suzuki, T. 2001. Influence of oocyte quality, culture media and gonadotrophins on cleavage rate and development of in vitro fertilized buffalo embryos. Anim. Reprod. Sci. 65: 215-223.

Ali, A. and Sirard, M. A. 2002. The effects of 17B-estradiol and protein supplement on the response to purified and recombinant follicle stimulating hormone in bovine oocytes. Zygote 10: 65-71.

Amer, H. A., Hegab, A. O. and Zaabal, S. M. 2008. Effects of ovarian morphology on oocyte quantity and quality, granulosa cells, in vitro maturation, and steroid hormone production in buffaloes. Anim Reprod 5: 55-62.

Anderson, M. 1985. Determination of glutathione and glutathione disulfide in biological samples. Methods Enzymol. 113: $548-555$.

Bavister, B. and Rose-Hellekant, T. 1992. Development of in vitro matured/in vitro fertilized bovine embryos into morulae and blastocysts in defined culture media. Theriogenology 37: 127-146.

Billaud, C. 2001. Composition, nutritional value and physiological properties. Adrian J. Fenugreek Sciences-des-ailment 21: $3-26$.

Cassidy, A. 2003. Potential risks and benefits of phytoestrogen-rich diets. Int J Vitam Nutr Res 73: 120-126.

Choi, Y. H., Carnevale, E. M., Siedel, G. E. and Squires, E. L. 2001. Effect of gonadotropins on bovine oocyte matured in TCM-199. Theriogenology 56: 661-670. 
Cognié, Y., Baril, G., Poulin, N. and Mermillod, P. 2003. Current status of embryo technologies in sheep and goat. Theriogenology 59: 171-188.

de Matos, D. G., Furnus, C. C., Moses, D. F., Martinez, A. G. and Matkovic, M. 1996. Stimulation of glutathione synthesis of in vitro matured bovine oocytes and its effect on embryo development and freezability. Mol. Reprod. Dev. 45: $451-457$.

de Matos, D. G. and Furnus, C. C. 2000. The importance of having high glutathione level after bovine in vitro maturation on embryo development: effect of $\beta$-mercaptoethanol, cysteine and cystine. Theriogenology 53: 761-771.

- and - 2007. The importance of having high glutathione (GSH) level after bovine in vitro maturation on embryo development: Effect of $\alpha$-mercaptoethanol, cystein and cystin. Theriogenology 53: 761-771.

-, Gasparrini, B., Pasqualini, S. R. and Thompson, J. G. 2002. Effect of glutathione synthesis stimulation during in vitro maturation of ovine oocytes on embryo development and intracellular peroxide content. Theriogenology $\mathbf{5 7}$ 1443-1451.

Dilip, M., Dola, M., Utpal, S., Sudipta, P. and Bhatta, C. 2006. In vitro effects of insulin-like growth factors and insulin on oocyte maturation and maturation-inducing steroid production in ovarian follicles of common carp, Cyprinus carpio. Comp. Biochem. Physiol. 144: 64-77.

Eppig, J. J. 1996. Coordination of nuclear and cytoplasmic oocyte maturation in eutherian mammals. Reprod. Fertil. Dev. 8 485-489.

Funahashi, H., Cantley, T. C., Stumpf, T. T. and Day, B. 1994. Use of low-salt culture medium with elevated oocytes glutathione levels. Biol. Rep. 51: 633-639.

Gasparrini, B., Sayoud, H., Neglia, G., de Matos, D. G. and Zicarelli, L. 2003. Glutathion synthesis during IVM of buffalo oocytes: effects of cysteamine on embryo development. Theriogenology 60: 943-952.

—, Boccia, L., Marchandise, J., Di Palo, R., George, F., Donnay, I. and Zicarelli, L. 2006. Enrichment of in vitro maturation medium for buffalo (Bubalus bubalis) oocytes with thiol compounds: effects of cystine on glutathione synthesis and embryo development. Theriogenology 65: 275-287.

Gray, S. L., Lackey, B. R., Tate, P. L. and Camper, N. D. 2004. Mycotoxins in root extracts of American and Asian Ginseng bind estrogen receptors. Exp. Bio. Med. 229: 560-568.

Guler, A., Poulin, N., Mermillod, P., Terqui, M. and Cognie, Y. 2000. Effect of growth factors, EGF and IGF-1 and estradiol on in vitro maturation of sheep oocytes. Theriogenology 54: 209-218.

Hassan, A. M. and Abdel-Wahhab, M. A. 2006. Antioxidant effect of parsley and panax ginseng extract against alteration induced in reproductive functions in male mice. Egypt J. Hosp. Med. 22: 60-72.

—, Khalil, W. K. B. and Ahmed, K. A. 2006. Genetic and histopathology studies on mice: effect of fenugreek oil on the efficiency of ovarian and liver tissues. Afr. J. Biotechnol. 5: 477-483.

Herrick, J. R., Behboodi, E., Memili, E., Blash, S., Echelard, Y. and Krisher, R. L. 2004. Effect of Macromolecule Supplementation during in Vitro Maturation of Goat Oocytes on Developmental Potential. Mol. Reprod. Develop. 69: 338-346.

Hibasami, H., Moteki, H. and Ishikawa, K. 2003. Protodioscin isolated from fenugreek (Trigonella foenum graecum L.) induces cell death and morphological change indicative of apoptosis in leukemic cell line $\mathrm{H}-60$, but not in gastric cancer cell line KATO III. Int. J. Mol. Med. 11: 23-26.

Higdon, J. V. and Frei, B. 2003. Polyphenols: health effects, metabolism and antioxidant functions. Crit Rev Food Sci Nutr 43: 89-143.

Hong, J. R., Yong, H. Y., Lee, B. C., Hwang, W. S., Lim, J. M. and Lee, E. S. 2004. Effects of amino acids on maturation, fertilization and embryo development of pig follicular oocytes in two IVM media. Theriogenology 62: 1473-1482.

Jamil, H., Samad, H. A., Rehman, N. U., Qureshi, Z. I. and Lodhi, L. A. 2007. In vitro maturation and fertilization of buffalo follicular oocytes in media supplemented with oestrus buffalo serum and hormones. Acta Vet. Brno 76: 399-404.

Jayaweera, D. M. A. 1981. Medicinal plant III. Peradeniya, Sri Lanka: Royal Botanic Garden, p. 255.

Kim, M. K., Lee, J. W., Lee, K. Y. and Yang, D. 2005. Microbial conversion of major ginsenoside Rbi to pharmaceutically active minor ginsenoside Rd. J. Microbiol. 43: 456-462.

Krisher, R. L. and Bavister, B. D. 1999. Enhanced glycolysis after maturation of bovine oocytes in vitro is associated with increased developmental competence. Mol. Reprod. Develop. 53: 19-26.

Lee, Y. J., Jin, Y. R., Lim, W. C., Park, W. K., Cho, J. Y., Jang, S. and Lee, S. K. 2003. Ginsenoside-Rb1 acts as a weak phytoestrogen in MCF-7 human breast cancer cells. Arch. Pharm. Res. 26: 58-63.

Li, Q., Niwa, K. and Hunter, M. G. 2004. Effects of 17B-Estradiol on in vitro maturation of pig oocytes in protein-free medium. J. Rep. Dev. 50: 305-313.

Miyamura, M., Yoshida, M., Hamano, S. and Kuwayama, M. 1995. Glutathione concentration during maturation and fertilization in bovine oocytes. Theriogenology 43: 282 .

Moor, R., Polge, C. and Wiladsen, S. 1980. Effect of follicular steroids on the maturation and fertilization of mammalian 
oocytes. J Embryol Exp Morphol 56: 319-335.

Niemann, H. and Rath, D. 2001. Progress in reproductive biotechnology in swine. Theriogenology 53: 1421-1437.

Norton, S. A. 1998. Useful plants of dermatology. III. Corticosteroids, strophanthus, and dioscorea. J. Am. Acad. Dermatol. 38: $256-259$.

Palop, V., Catalan, C., Rubio, E. and Martinez-Mir, I. 1999. Gynecomastia in a male and ginseng. Med Clin 112: 758.

Palta, P. and Chauhan, M. S. 1998. Laboratory production of buffalo (Bubalus bubalis) embryos. Reprod. Fertil. Dev. 10: 379-391.

Pawshe, C., Palanisamy, A., Taneja, M., Jain, S. K. and Totey, S. M. 1996. Comparison of various maturation treatments on in vitro maturation of goat oocytes and their early embryonic development and cell numbers. Theriogenology 46: 971-981.

Petit, P. R., Sauviaire, Y. D., Hillaire-Buys, D. M., Leconte, O. M. and Baissac, Y. G. 1995. Steroid saponins from fenugreek seeds: extraction, purification and pharmacological investigation on feeding behavior and plasma cholesterol. Steroids 10: 674-680.

Rizos, D., Lonergan, P., Boland, M. P., Arroyo-Garcia, R., Pintado, B., Dela-fuente, J. and Gutierrez-Adan, A. 2002. Analysis of differential messenger RNA expression between bovine blastocyst produce in different culture system: implication for blastocyst quality. Biol. Reprod. 66: 589-595.

Rosenkrans, C. R. and First, N. L. 1994. Effect of free amino acids and vitamins on cleavage and development rate of bovine zygotes in vitro. J. Anim. Sci. 72: 434-437.

Roushandeh, A. M., Pasbakhsh, P., Alizadeh, Z. and Roudkenar, M. H. 2007. In vitro maturation media, cysteamine concentration and glutathione level affect blstocysts development in mouse. Iranian J. Reprod. Med. 5: 159-163.

Ruth, R., Franks, S. and Hardy, K. 2002. Culture environment modulates maturation and metabolism of human oocytes. Hum. Reprod. 17: 2950-2956.

Salvati, G., Genovesi, G., Marcellini, L., Paolini, P., De, N. I., Pepe, M. and Re, M. 1996. Effects of Panax ginseng C.A. Meyer saponins on male fertility. Panminerva Med 38: 249-254.

Santos, L. C., Rodrigues, B. A. and Rodrigues, J. L. 2006. In vitro nuclear maturation of bitch oocytes in the presence of polyvinyl-pyrrolidone. Anim Reprod 3: 70-75.

Sharma, R. D., Sarkar, A. and Hazra, D. K. 1996. Hypolipidaemic effect of fenugreek seeds. Phytother. Res. 10: $332-334$.

Smetanina, I. G., Tatarinova, L. V. and Krivokharchenko, A. S. 2000. The effect of the composition of the culture media on bovine oocyte maturation and embryo development in vitro. Ontogenez. 31: 139-143.

Sur, P., Das, M. and Gomes, A., 2001. Trigonella foenum graecum (fenugreek) seed extract as an antineoplastic agent. Phytother. Res. 15: 257-259.

Tesarik, J. and Mendoza, C. 1997. Direct non-genomic effects of follicular steroids on maturing human oocytes. Hum. Rep. Update 3: 95-100.

Tsai, S. C., Chiao, Y. C., Lu, C. C. and Wang, P. S. 2003. Stimulation of the secretion of luteinizing hormone by ginsenoside-Rb1 in male rat. Chin J Physiol 46: 1-7.

Wang, Z., Yu, S. and $\mathrm{Xu}, \mathrm{Z}$. 2007. Improvement in bovine embryo production in vitro by treatment with green tea polyphenols during in vitro maturation of oocytes. Anim. Rep. Sci. 100: 22-31.

Yoshida, M., Ishigaki, K., Nagai, T., Chikyu, M. and Pursel, V. G. 1993. Glutathione concentration during maturation and after fertilization in pig oocytes: relevance to the ability of oocytes to form male pronucleus. Biol. Reprod. 49: 89-94.

Yoshida, M. Y., Ishizaki, K., Kawagishi, H., Bamba, K. and Kojima, Y. 1992. Effects of pig follicular fluid on maturation of pig oocytes in vtiro and on their subsequent fertilizing and developmental capacity in vitro. J. Reprod. Fertil. 95: 481-488.

Zuelke, K. A., Jeffay, S. C., Zucker, R. M. and Perreault, S. D. 2003. Glutathione (GSH) concentrations vary with the cell cycle in maturing hamster oocytes, zygotes, and pre-implantation stage embryos. Mol. Reprod. Dev. 64: 106-112. 